\title{
SUFFICIENT CONDITION FOR THE EXISTENCE OF SOLUTIONS OF A FREE BOUNDARY PROBLEM
}

\author{
BY \\ MOHAMMED HAYOUNI (Institut Elie Cartan, B.P. 239, 54506 VandEuvre-lès-Nancy, France) \\ AND \\ ARIAN NOVRUZI (Institut Elie Cartan, B.P. 239, 54506 VandEuvre-lès-Nancy, France)
}

\begin{abstract}
In this paper we study the existence of critical points of a functional depending on $\Omega \subset \mathbb{R}^{2}$ through its perimeter and the solution of the Dirichlet problem in $\Omega$, under the constraint that the measure of $\Omega$ is given. We give a sufficient condition for the existence of critical points using the implicit function theorem.
\end{abstract}

1. Introduction. In this paper we present a sufficient condition for the existence of regular critical points of some energy functional associated with an interior twodimensional electromagnetic casting problem. This problem has been studied from different points of view by some authors, for instance [1], [3], [4], [6]. However, the existence of critical points of the energy functional we consider (which depends on the solution of a Dirichlet problem and the perimeter) is still an open problem.

Let $p>2, j \in \mathbb{L}^{p}\left(\mathbb{R}^{2}\right)$. For any $\Omega \subset \mathbb{R}^{2}$, an open bounded subset, let $m(\Omega)$ be its Lebesgue measure and $u_{\Omega}$ the variational solution in $H_{0}^{1}(\Omega)$ of the following interior Dirichlet problem:

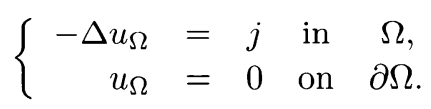

Let $m_{0}, \mu$ be two prescribed positive numbers. We consider the problem of finding a $C^{2}$ bounded open subset $\Omega^{*}$ of $\mathbb{R}^{2}$ and $\Lambda^{*} \in \mathbb{R}$ such that

$$
\left\{\begin{array}{l}
-\frac{1}{2}\left(\frac{\partial u_{\Omega^{*}}}{\partial \nu^{*}}\right)^{2}+\mu \mathcal{H}^{*}+\Lambda^{*}=0 \text { on } \partial \Omega^{*} \\
m\left(\Omega^{*}\right)-m_{0}=0
\end{array}\right.
$$

where $\mathcal{H}^{*}$ is the curvature of $\partial \Omega^{*}$ and $\nu^{*}$ is the unit exterior normal vector to $\partial \Omega^{*}$.

Received May 4, 1999.

2000 Mathematics Subject Classification. Primary 62K05; 49K30.

E-mail address: hayouni@iecn.u-nancy.fr

E-mail address: novruzi@iecn.u-nancy.fr

(C)2002 Brown University 
Equations (2) are necessary conditions for a regular set $\Omega^{*}$ to be a solution of the following shape optimization problem (see, for example, [4]):

$$
E\left(\Omega^{*}\right)=\min \{E(\Omega), \Omega \in \mathcal{O}\},
$$

where $\mathcal{O}=\left\{\Omega \subset \mathbb{R}^{2}, C^{2}\right.$ bounded open set and $\left.m(\Omega)=m_{0}\right\}$,

$$
e(\Omega)=-\frac{1}{2} \int_{\Omega}\left|D u_{\Omega}(y)\right|^{2} d y, \quad P(\Omega)=\int_{\partial \Omega} d \partial \Omega,
$$

with $D=\left(\partial_{x}, \partial_{y}\right)$, and $E(\Omega)=e(\Omega)+P(\Omega)$. Recall that $e(\Omega)$ is nothing but the "energy" of the Dirichlet problem in $\Omega$.

The regular subset $\Omega^{*}$ that obeys (2) is called a critical point of $E$ under the constraint $m(\Omega)=m_{0}$.

In Theorem 1.1 we give a sufficient condition for the existence of critical points of $E$ for $\mu$ large enough. The idea for such a condition is suggested by the following consideration. Let us assume :

(i) for any $\mu>>1$, there exists $\Omega_{\mu}^{*}$ solution of (3);

(ii) there exists $\Omega^{*} \in \mathcal{O}$ such that

$$
e\left(\Omega_{\mu}^{*}\right) \underset{\mu \rightarrow \infty}{\longrightarrow} e\left(\Omega^{*}\right) \text { with } e\left(\Omega^{*}\right) \text { finite and } P\left(\Omega_{\mu}^{*}\right) \underset{\mu \rightarrow \infty}{\longrightarrow} P\left(\Omega^{*}\right) .
$$

Since $m\left(\Omega_{\mu}^{*}\right)=m_{0}$, it follows by the isoperimetric inequality that $P\left(\Omega_{\mu}^{*}\right)=2 \pi r_{0}+\epsilon_{\mu}$ with $\epsilon_{\mu} \geq 0$ and $r_{0}=\left(m_{0} / \pi\right)^{1 / 2}$. From (i) we have $E\left(\Omega_{\mu}^{*}\right) \leq E\left(B\left(x, r_{0}\right)\right)$, for any $x \in \mathbb{R}^{2}$, where $B(x, r)$ is the ball with radius $r$ centered at $x$. Thus

$$
e\left(\Omega_{\mu}^{*}\right)+\mu \epsilon_{\mu} \leq e\left(B\left(x, r_{0}\right)\right), \quad \forall x \in \mathbb{R}^{2} .
$$

From (ii) we deduce that $\lim _{\mu \rightarrow+\infty} \epsilon_{\mu}=0$ and therefore $P\left(\Omega^{*}\right)=2 \pi r_{0}$ and $m\left(\Omega^{*}\right)=\pi r_{0}^{2}$; this implies that $\Omega^{*}=B\left(x_{0}, r_{0}\right)$ for some $x_{0} \in \mathbb{R}^{2}$. Taking $x=x_{0}$ in (5) and letting $\mu$ tend to infinity, we get $\lim _{\mu \rightarrow \infty} \mu \epsilon_{\mu}=0$. Then, from (5) we have (letting $\mu$ tend to infinity): $e\left(B\left(x_{0}, r_{0}\right)\right) \leq e\left(B\left(x, r_{0}\right)\right)$, for $x \in \mathbb{R}^{2}$, which says that $x_{0}$ is a minimum of the function

$$
h(x):=-\frac{1}{2} \int_{B\left(x, r_{0}\right)}\left|D u_{B\left(x, r_{0}\right)}(y)\right|^{2} d y .
$$

Now, since we are interested in the solutions of (2), we shall assume that there exists $x_{0} \in \mathbb{R}^{2}$ such that

$$
D h\left(x_{0}\right)=(0,0), \quad D^{2} h\left(x_{0}\right) \text { has nonzero eigenvalues, }
$$

where $D^{2}$ is the matrix operator $D^{t} \cdot D$.

Thus, we think of the solutions $\Omega^{*}$ of $(2)$ as perturbations of the domain $\Omega_{0}:=$ $B\left(x_{0}, r_{0}\right)$ (letting $\Gamma_{0}$ denote the boundary of $\left.\Omega_{0}\right)$. Since $\mu>>1$, we set $\mu=\sigma^{-2}$ and introduce

$$
T:\left\{\begin{array}{lll}
\mathbb{R} \times C^{2}\left(\Gamma_{0}\right) & \longrightarrow & C^{2}\left(\Gamma_{0} ; \mathbb{R}^{2}\right), \\
(\sigma, \psi) & \longmapsto & I+U(\sigma, \psi):=I+t(\psi)+\sigma \psi \nu_{0},
\end{array}\right.
$$

where $I$ is the identity on $\mathbb{R}^{2}, \nu_{0}$ is the unit exterior normal vector to $\Gamma_{0}$ and $t(\psi)=$ $f_{\Gamma_{0}} \psi \cdot \nu_{0} d s_{0}:=\frac{1}{\left|\Gamma_{0}\right|} \int_{\Gamma_{0}} \psi \cdot \nu_{0} d s_{0}$, with $s_{0}$ the arclength of $\Gamma_{0}$. We let $\Gamma(\sigma, \psi)=\{T(\sigma, \psi)(x)$, $\left.x \in \Gamma_{0}\right\}$, where $\Omega(\sigma, \psi)$ denotes the interior domain defined by $\Gamma(\sigma, \psi), \nu(\sigma, \psi)$ denotes 
the unit exterior normal vector to $\Gamma(\sigma, \psi), \mathcal{H}(\sigma, \psi)$ denotes the curvature of $\Gamma(\sigma, \psi), \mathcal{H}_{0}$ denotes the curvature of $\Gamma_{0}, \mathbf{u}(\sigma, \psi)=u_{\Omega(\sigma, \psi)}$ and $\mathbf{m}(\sigma, \psi)=m(\Omega(\sigma, \psi))$.

The main result we prove is:

THEOREM 1.1. Under the hypothesis (6) there exist $\sigma^{*}>0$ and a unique $C^{1}$ map $\Sigma$ : $\left(-\sigma^{*}, \sigma^{*}\right) \longmapsto C^{2}\left(\Gamma_{0}\right) \times \mathbb{R}$ such that for any $\sigma \in\left(-\sigma^{*}, \sigma^{*}\right) \backslash\{0\}$ if $\Sigma(\sigma)=(\psi, \Lambda)$ we have

$$
\begin{aligned}
& -\frac{1}{2}\left(\frac{\partial \mathbf{u}(\sigma, \psi)}{\partial \nu(\sigma, \psi)}\right)^{2}+\frac{1}{\sigma^{2}} \mathcal{H}(\sigma, \psi)+\left(\frac{\Lambda}{\sigma}-\frac{\mathcal{H}_{0}}{\sigma^{2}}\right)=0 \text { on } \Gamma(\sigma, \psi), \\
& \mathbf{m}(\sigma, \psi)-m_{0}=0 .
\end{aligned}
$$

REMARK 1.2. The main tool we use to prove existence of critical points of $E$ is the implicit function theorem applied near $(0,0,0)$ to some function $F$ of $(\sigma, \psi, \Lambda)$ (see (17), (18)), which involves the left-hand side of $(7)$.

The hypothesis (6) is not explicit. However, in the case of radial functions, this hypothesis is equivalent to another explicit condition.

Proposition 1.3. Assume that $j \in \mathbb{L}^{p}\left(\mathbb{R}^{2}\right)$ is radial and $u_{\Omega_{0}} \in W^{3,1}\left(\Omega_{0}\right)$. Then for $x_{0}=(0,0)$, the condition $(6)$ is equivalent to

$$
\left(j\left(r_{0}\right)-\frac{2}{r_{0}^{2}} \int_{0}^{r_{0}} j(r) r d r\right) \int_{0}^{r_{0}} j(r) r d r \neq 0 .
$$

Thus, in particular, for any radial function $j$ with $\operatorname{supp}(j) \subset B\left(0, r_{0}\right)$ and integral nonzero (with respect to the measure $r d r$ ) the hypotheses (6) are always fulfilled.

Proof. Using classical results concerning the differentiability with respect to the shape of an integral which depend on the solution $u_{\Omega}$ (see, for example, [7], [8]) we can prove that

$$
\begin{aligned}
D h(0) & =-\frac{1}{2} \int_{\Gamma_{0}}\left(\frac{\partial u_{\Omega_{0}}}{\partial \nu_{0}}\right)^{2} \nu_{0} d s_{0} \\
D_{i, j}^{2} h(0) & =-\int_{\Gamma_{0}} \frac{\partial u_{\Omega_{0}}}{\partial \nu_{0}} \frac{\partial u_{\Omega_{0}}^{\prime}\left(\nu_{0}^{j}\right)}{\partial \nu_{0}} \nu_{0}^{i}+\left(\frac{\partial u_{\Omega_{0}}}{\partial \nu_{0}}\right)^{2} \frac{2 \nu_{0}^{i} \nu_{0}^{j}-\delta_{i j}}{2 r_{0}}+\frac{\partial u_{\Omega_{0}}}{\partial \nu_{0}} \frac{\partial^{2} u_{\Omega_{0}}}{\partial \nu_{0}^{2}} \nu_{0}^{i} \nu_{0}^{j},
\end{aligned}
$$

where $u_{\Omega_{0}}^{\prime}\left(\nu_{0}^{j}\right)$ is the shape derivative of $u_{\Omega}$ at $\Omega_{0}$ in the direction $\nu_{0}^{j}$, given by

$$
\left\{\begin{array}{rccc}
-\Delta u_{\Omega_{0}}^{\prime}\left(\nu_{0}^{j}\right) & = & 0 & \text { in } \quad \Omega_{0}, \\
u_{\Omega_{0}}^{\prime}\left(\nu_{0}^{j}\right) & = & -\nu_{0}^{j} \frac{\partial u_{\Omega_{0}}}{\partial \nu_{0}} & \text { on } \quad \Gamma_{0},
\end{array}\right.
$$

with $i, j=1,2$ and $\nu_{0}=\left(\nu_{0}^{1}, \nu_{0}^{2}\right)$. We remark that, with the hypothesis of the proposition, the condition of the proposition, $D h(0)$ and $D^{2} h(0,0)$ are well defined.

It is easy to see that when $j$ is a radial function we have that

$$
u_{\Omega_{0}}(y)=-\int_{0}^{|y|} \frac{1}{s} \int_{0}^{s} j(r) r d r+\int_{0}^{r_{0}} \frac{1}{s} \int_{0}^{s} j(r) r d r, \quad u_{\Omega_{0}}^{\prime}\left(\nu_{0}^{i}\right)(y)=-\frac{y_{i}}{r_{0}} \frac{\partial u_{\Omega_{0}}}{\partial \nu_{0}} .
$$

It is clear that for $|y|=r_{0}$ we also have

$$
\frac{\partial u_{\Omega_{0}}}{\partial \nu_{0}}(y)=-\frac{1}{r_{0}} \int_{0}^{r_{0}} j(r) r d r, \quad \frac{\partial^{2} u_{\Omega_{0}}}{\partial \nu_{0}^{2}}(y)=\frac{1}{r_{0}^{2}} \int_{0}^{r_{0}} j(r) r d r-j\left(r_{0}\right) .
$$


Then we obtain these expressions for $D h(0)$ and $D^{2} h(0)$ :

$$
\begin{aligned}
D h(0) & =(0,0) \\
D^{2} h(0) & =\pi\left[\left(\frac{\partial u_{\Omega_{0}}}{\partial \nu_{0}}\right)^{2}-r_{0} \frac{\partial u_{\Omega_{0}}}{\partial \nu_{0}} \frac{\partial^{2} u_{\Omega_{0}}}{\partial \nu_{0}^{2}}\right]\left(\begin{array}{cc}
1 & 0 \\
0 & 1
\end{array}\right) \\
& =\pi\left[\frac{2}{r_{0}^{2}}\left(\int_{0}^{r_{0}} j(r) r d r\right)^{2}-j\left(r_{0}\right) \int_{0}^{r_{0}} j(r) r d r\right]\left(\begin{array}{ll}
1 & 0 \\
0 & 1
\end{array}\right)
\end{aligned}
$$

which prove the first part of the proposition. The second part follows immediately because when $\sup (j) \subset B\left(0, r_{0}\right)$, we have $j\left(r_{0}\right)=0$.

REMARK 1.4. When the conditions of Proposition 1.3 are not satisfied, typically when $D^{2} h(0,0)$ is not invertible, the result of Theorem 1.1 fails. However, this does not mean that there are not optimal shapes but, probably, there is no uniqueness.

For example, let us consider $j(y)=1$ in $D_{\epsilon}:=\left\{|y| \leq r_{0}+\epsilon\right\}$. The condition of Proposition 1.3 fails $\left(D^{2} h(0)\right.$ is not invertible). However, the disk $B\left(0, r_{0}\right)$, which obviously is an optimal shape, is not unique. Indeed, for any $x \in D_{\epsilon}$ we have that $u_{B\left(x, r_{0}\right)}$ is equal to $u_{B\left(0, r_{0}\right)}$, modulo one translation. Thus, the optimal shape $B\left(0, r_{0}\right)$ is not unique.

2. Preliminary results. Let $\tau_{0}$ (resp. $\tau(\sigma, \psi)$ ) denote the unit tangent vector to $\Gamma_{0}$ (resp. $\Gamma(\sigma, \psi))$ such that $\nu_{0} \wedge \tau_{0}=1$ (resp. $\nu(\sigma, \psi) \wedge \tau(\sigma, \psi)=1$ ) where $\wedge$ is the product operator given by

$$
x \wedge y=x_{1} y_{2}-x_{2} y_{1}, \text { where } x=\left(x_{1}, x_{2}\right), y=\left(y_{1}, y_{2}\right) .
$$

Let $s(\sigma, \psi)$ be the arclength of $\Gamma(\sigma, \psi)$. We recall that $\Gamma(\sigma, \psi)=\left\{T(\sigma, \psi)(x), x \in \Gamma_{0}\right\}$. Then we have

$$
\begin{aligned}
\frac{d}{d s_{0}}[s(\sigma, \psi) \circ T(\sigma, \psi)] & =\left|\frac{d}{d s_{0}} T(\sigma, \psi)\right|=\left|\tau_{0}+\sigma \frac{d\left(\psi \nu_{0}\right)}{d s_{0}}\right| \\
& =\left(1+2 \sigma \mathcal{H}_{0} \psi+\sigma^{2}\left[\mathcal{H}_{0}^{2} \psi^{2}+\left(\frac{d \psi}{d s_{0}}\right)^{2}\right]\right)^{1 / 2},
\end{aligned}
$$

where $d(\cdot) / d s_{0}$ is the derivative of $(\cdot)$ with respect to the arclength of $\Gamma_{0}$.

In order to simplify the notation when there is no confusion, we do not specify the dependence on $(\sigma, \psi)$. Thus, we will write just $\Gamma, \Omega, \nu, \tau, \mathcal{H}, \mathbf{u}, \mathbf{m}, T, U$ and $s$ instead of $\Gamma(\sigma, \psi), \Omega(\sigma, \psi), \nu(\sigma, \psi), \tau(\sigma, \psi), \mathcal{H}(\sigma, \psi), \mathbf{u}(\sigma, \psi), \mathbf{m}(\sigma, \psi), T(\sigma, \psi), U(\sigma, \psi)$ and $s(\sigma, \psi)$.

Lemma 2.1. The application $(\sigma, \psi) \longmapsto(\nu \circ T, \mathcal{H} \circ T)$ from $\mathbb{R} \times C^{2}\left(\Gamma_{0}\right)$ into $C^{0}\left(\Gamma_{0} ; \mathbb{R}^{2}\right) \times$ $C^{0}\left(\Gamma_{0}\right)$ is of class $C^{1}$ near $(0,0)$.

Moreover, the map $(\sigma, \psi) \longmapsto \frac{\mathcal{H}(\sigma, \psi) \circ T(\sigma, \psi)-\mathcal{H}_{0}}{\sigma}$ from $\mathbb{R} \times C^{2}\left(\Gamma_{0}\right)$ into $C^{0}\left(\Gamma_{0}\right)$ is of class $C^{1}$ near $(0,0)$ and

$$
D_{\psi} \frac{\mathcal{H}(\sigma, \psi) \circ T(\sigma, \psi)-\mathcal{H}_{0}}{\sigma}(0,0)(\phi)=-\frac{d^{2} \phi}{d s_{0}^{2}}-\mathcal{H}_{0}^{2} \phi .
$$


Proof. To prove the $C^{1}$ regularity of $\nu \circ T$, it suffices to prove the $C^{1}$ regularity of $\tau \circ T$, because $\nu \circ T$ is obtained by a rotation of $\tau \circ T$. It is clear that the vector $d T / d s_{0}$ is tangent to $\Gamma$. Then

$$
\begin{aligned}
\tau \circ T & =\frac{d T}{d s_{0}}\left|\frac{d T}{d s_{0}}\right|^{-1}=\frac{d\left(I+t(\psi)+\sigma \psi \nu_{0}\right)}{d s_{0}}\left|\frac{d\left(I+t(\psi)+\sigma \psi \nu_{0}\right)}{d s_{0}}\right|^{-1} \\
& =\left(\tau_{0}+\sigma \frac{d\left(\psi \nu_{0}\right)}{d s_{0}}\right)\left(\frac{d(s \circ T)}{d s_{0}}\right)^{-1}
\end{aligned}
$$

The $C^{1}$ regularity of $\tau \circ T$ near $(0,0)$ is clear, because $\tau_{0}+\sigma d\left(\psi \nu_{0}\right) / d s_{0}$ is of class $C^{1}$ from $\mathbb{R} \times C^{2}\left(\Gamma_{0}\right)$ into $C^{0}\left(\Gamma_{0}\right)$. From (9) one can see that $\left(\frac{d(s \circ T)}{d s_{0}}\right)^{-1}$ is of class $C^{1}$ in a small neighbourhood of $(0,0)$ in $\mathbb{R} \times C^{2}\left(\Gamma_{0}\right)$.

On the other hand, the curvature of a parametric curve is given by

$$
\mathcal{H} \circ T=\frac{d T}{d s_{0}} \wedge \frac{d^{2} T}{d s_{0}^{2}}\left(\frac{d(s \circ T)}{d s_{0}}\right)^{-3} .
$$

This formula shows the $C^{1}$ regularity of $\mathcal{H} \circ T$ near $(0,0)$, because $d T / d s_{0}$ and $d^{2} T / d s_{0}^{2}$ are of class $C^{1}$ from $\mathbb{R} \times C^{2}\left(\Gamma_{0}\right)$ into $C^{0}\left(\Gamma_{0}\right)$ and, thanks to $(9),\left(\frac{d(s \circ T)}{d s_{0}}\right)^{-3}$ is of class $C^{1}$ in a small neighbourhood of $(0,0)$ in $\mathbb{R} \times C^{2}\left(\Gamma_{0}\right)$. This proves the first statement of the lemma.

On the other hand, using (12) we can write

$$
\begin{aligned}
\frac{\mathcal{H}(\sigma, \psi) \circ T(\sigma, \psi)-\mathcal{H}_{0}}{\sigma}= & \frac{1}{\sigma}\left(\frac{d T}{d s_{0}} \wedge \frac{d^{2} T}{d s_{0}^{2}}-\mathcal{H}_{0}\right)\left(\frac{d(s \circ T)}{d s_{0}}\right)^{-3} \\
& +\frac{\mathcal{H}_{0}}{\sigma}\left(\left(\frac{d(s \circ T)}{d s_{0}}\right)^{-3}-1\right) \\
\frac{1}{\sigma}\left(\frac{d T}{d s_{0}} \wedge \frac{d^{2} T}{d s_{0}^{2}}-\mathcal{H}_{0}\right)= & \mathcal{H}_{0}^{2} \psi\left(2+\sigma \mathcal{H}_{0} \psi\right) \\
& +2 \sigma \mathcal{H}_{0}\left(\frac{d \psi}{d s_{0}}\right)^{2}-\left(1+\sigma \mathcal{H}_{0} \psi\right) \frac{d^{2} \psi}{d s_{0}^{2}} .
\end{aligned}
$$

Then, the second statement of the lemma follows easily using (9).

For $(\sigma, \psi) \in \mathbb{R} \times C^{2}\left(\Gamma_{0}\right)$, let $\hat{U}(\sigma, \psi)$ be a $C^{2}$ extension with compact support of $U(\sigma, \psi)$ given, for example, by Lemma 6.38 in [2], and $\hat{T}(\sigma, \psi)=I+\hat{U}(\sigma, \psi)$. Let $D \hat{T}(\sigma, \psi)$ (resp. $[D \hat{T}(\sigma, \psi)]^{-1}$ ) denote the Jacobian matrix of $\hat{T}(\sigma, \psi)$ (resp. the inverse matrix of $D \hat{T}(\sigma, \psi))$. From [8] we have that

$$
(\sigma, \psi) \longmapsto\left(D \hat{T}(\sigma, \psi),[D \hat{T}(\sigma, \psi)]^{-1}\right)
$$

from $\mathbb{R} \times C^{2}\left(\Gamma_{0}\right)$ into $C^{0}\left(\mathbb{R}^{2} ; \mathbb{R}^{4}\right) \times C^{0}\left(\mathbb{R}^{2} ; \mathbb{R}^{4}\right)$ is of class $C^{1}$ near $(0,0)$.

From [8], essentially using the implicit function theorem, we also have

Lemma 2.2. The function $(\sigma, \psi) \longmapsto \mathbf{u} \circ \hat{T}$ from $\mathbb{R} \times C^{2}\left(\Gamma_{0}\right)$ into $W^{2, p}\left(\Omega_{0}\right)$ is of class $C^{1}$ near $(0,0)$. 
Corollary 2.3. For $p>2$ the function $(\sigma, \psi) \longmapsto \frac{\partial \mathbf{u}}{\partial \nu} \circ T$ from $\mathbb{R} \times C^{2}\left(\Gamma_{0}\right)$ into $C^{0}\left(\Gamma_{0}\right)$ is of class $C^{1}$ near $(0,0)$.

Proof. For $p>2$ the inclusion $W^{2, p}\left(\Omega_{0}\right) \subset C^{1}\left(\Omega_{0}\right)$ is continuous. It follows from Lemma 2.2 that $(\sigma, \psi) \longmapsto \mathbf{u} \circ \hat{T}$ is of class $C^{1}$ near $(0,0)$, from $\mathbb{R} \times C^{2}\left(\Gamma_{0}\right)$ into $C^{1}\left(\Omega_{0}\right)$. Thus, $(\sigma, \psi) \longmapsto D(\mathbf{u} \circ \hat{T})$ is of class $C^{1}$ near $(0,0)$ from $\mathbb{R} \times C^{2}\left(\Gamma_{0}\right)$ into $\left(C^{0}\left(\Omega_{0}\right)\right)^{3}$. But

$$
\frac{\partial \mathbf{u}}{\partial \nu} \circ T=\nu \circ T \cdot[\hat{T}]^{-1} \cdot D(\mathbf{u} \circ \hat{\mathrm{T}}) \text { on } \Gamma_{0}
$$

This equality and the continuity of the trace operator from $C^{0}\left(\Omega_{0}\right)$ into $C^{0}\left(\Gamma_{0}\right)$ prove the corollary.

Set $\mathbf{e}(\sigma, \psi)=e(\Omega(\sigma, \psi))$. We give the next lemma without proof, but we note that it follows from the differentiability of $(\sigma, \psi) \longmapsto \hat{T}$ from $\mathbb{R} \times C^{2}\left(\Gamma_{0}\right)$ into $C^{2}\left(\Gamma_{0} ; \mathbb{R}^{2}\right)$ and Lemma 2.2 .

Lemma 2.4. The application $(\sigma, \psi) \in \mathbb{R} \times C^{2}\left(\Gamma_{0}\right) \longmapsto \mathbf{e}(\sigma, \psi) \in \mathbb{R}$ is of class $C^{1}$ near $(0,0)$. Moreover, $D_{\psi} \mathbf{e}(\sigma, \psi)(\varphi)$, the derivative of $\mathbf{e}$ with respect to $\psi$ at $(\sigma, \psi)$ in the direction $\varphi$, is given by

$$
D_{\psi} \mathbf{e}(\sigma, \psi)(\varphi)=-\frac{1}{2} \int_{\Gamma}\left(\frac{\partial \mathbf{u}}{\partial \nu}\right)^{2}\left(U(\sigma, \varphi) \circ T^{-1} \cdot \nu\right) d s
$$

LEMma 2.5. The application $(\sigma, \psi) \in \mathbb{R} \times C^{2}\left(\Gamma_{0}\right) \longmapsto \mathbf{e}(\sigma, \psi)(\varphi) \in \mathbb{R}$ is of class $C^{2}$ near $(0,0)$. Moreover, $D_{\psi}^{2} \mathbf{e}(0,0)(\varphi, \phi)$, the second derivative of $\mathbf{e}$ with respect to $\psi$ at $(0,0)$ in the direction $(\varphi, \phi)$, is given by

$$
D_{\psi}^{2} \mathbf{e}(0,0)(\varphi, \phi)=-\frac{1}{2} \int_{\Gamma_{0}}\left(t(\varphi) \cdot \nu_{0}\right) D_{\psi}\left[\left(\frac{\partial \mathbf{u}}{\partial \nu}\right)^{2} \circ T\right](0,0)(\phi) d s_{0} .
$$

Proof. We change the variable in the expression of $D_{\psi} \mathbf{e}(\sigma, \psi)(\varphi)$ in order to have an integral on $\Gamma_{0}$ :

$$
D_{\psi} \mathbf{e}(\sigma, \psi)(\varphi)=-\frac{1}{2} \int_{\Gamma_{0}}\left(\frac{\partial \mathbf{u}}{\partial \nu}\right)^{2} \circ T(U(\sigma, \varphi) \cdot \nu \circ T) \frac{d(s \circ T)}{d s_{0}} d s_{0} .
$$

But $U(\sigma, \varphi)=t(\varphi)+\sigma \varphi \nu_{0}$ implies

$$
\begin{aligned}
D_{\psi} \mathbf{e}(\sigma, \psi)(\varphi)= & -\frac{\sigma}{2} \int_{\Gamma_{0}}\left(\frac{\partial \mathbf{u}}{\partial \nu}\right)^{2} \circ T\left(\varphi \nu_{0} \cdot \nu \circ T\right) \frac{d(s \circ T)}{d s_{0}} d s_{0} \\
& -\frac{1}{2} \int_{\Gamma_{0}}(t(\varphi) \cdot \nu \circ T)\left[\left(\frac{\partial \mathbf{u}}{\partial \nu}\right)^{2} \circ T\right] \frac{d(s \circ T)}{d s_{0}} d s_{0} .
\end{aligned}
$$

The existence of a second-order differential of e near $(0,0)$ follows from Lemma 2.1 and Corollary 2.3. On the other hand, $\nu \circ T$ is obtained by a rotation of $\tau \circ T$. Then from (11):

$$
D_{\psi}\left(\tau \circ T \frac{d(s \circ T)}{d s_{0}}\right)(0,0)(\phi)=D_{\psi}\left(\tau_{0}+\sigma \frac{d\left(\psi \nu_{0}\right)}{d s_{0}}\right)(0,0)(\phi)=0 .
$$

Consequently, $D_{\psi}\left(\nu \circ T \frac{d s \circ T}{d s_{0}}\right)(0,0)=0$ and, by differentiating (15), we get the expression (14). 
REMARK 2.6. It is clear that $T(0, \psi)\left(\Omega_{0}\right)=(I+t(\psi))\left(\Omega_{0}\right)=B\left(x_{0}+t(\psi), r_{0}\right)$. Thus, $\mathbf{e}(0, \psi)=h\left(x_{0}+t(\psi)\right)$ and, consequently,

$$
D_{\psi}^{2} \mathbf{e}(0,0)(\varphi, \phi)=t(\varphi) \cdot D^{2} h\left(x_{0}\right) \cdot t(\phi) .
$$

Let $F$ be the following function:

$$
\begin{aligned}
F: \mathbb{R} \times C^{2}\left(\Gamma_{0}\right) \times \mathbb{R} & \longmapsto C^{0}\left(\Gamma_{0}\right) \times \mathbb{R}, \\
(\sigma, \psi, \Lambda) & \longmapsto\left(F_{1}(\sigma, \psi, \Lambda), F_{2}(\sigma, \psi, \Lambda)\right),
\end{aligned}
$$

where $F_{1}, F_{2}$ are given by

$$
\begin{aligned}
F_{1}(\sigma, \psi, \Lambda)= & -\frac{\nu_{0}}{2\left|\Gamma_{0}\right|} \cdot \int_{\Gamma}\left(\frac{\partial \mathbf{u}(\sigma, \psi)}{\partial \nu(\sigma, \psi)}\right)^{2} \nu(\sigma, \psi) d s-\frac{\sigma}{2}\left(\frac{\partial \mathbf{u}(\sigma, \psi)}{\partial \nu(\sigma, \psi)}\right)^{2} \circ T \\
& +\frac{\mathcal{H}(\sigma, \psi) \circ T(\sigma, \psi)-\mathcal{H}_{0}}{\sigma}+\Lambda, \\
F_{2}(\sigma, \psi, \Lambda)= & (\mathbf{m}(\sigma, \psi)-\mathbf{m}(0, \psi)) / \sigma .
\end{aligned}
$$

REMARK 2.7. Note that $\sigma \cdot F_{2}$ is the left-hand side (l.h.s.) of (8) and that $F_{1} / \sigma$ differs from the l.h.s. of $(7)$ by $-\frac{\nu_{0}}{2\left|\Gamma_{0}\right|} \cdot \int_{\Gamma}\left(\frac{\partial \mathbf{u}(\sigma, \psi)}{\partial \nu(\sigma, \psi)}\right)^{2} \nu(\sigma, \psi) d s$.

LEMmA 2.8. For $\psi \in C^{2}\left(\Gamma_{0}\right)$ and $\sigma \neq 0$ sufficiently small, the equation $F(\sigma, \psi, \Lambda)=0$ is equivalent to $(7)$ and $(8)$.

Proof. From the above remark, to prove the lemma it suffices to show that $F_{1}(\sigma, \psi, \Lambda)$ $=0$ is equivalent to $(7)$.

Assume that (7) is satisfied and $\nu=\left(\nu^{1}, \nu^{2}\right)$. For $\varphi \in C^{2}\left(\Gamma_{0}\right)$ it is easy to show that

$$
\int_{\Gamma} \mathcal{H}(\sigma, \psi) \nu^{i} d s=0 \quad \text { and } \quad \int_{\Gamma} \nu^{i} d s=0, \quad i=1,2 .
$$

Note that the first equality above is obtained from the formula of integration by parts on the boundary $\Gamma_{0}$ (see, for instance, [2]).

Then, multiplying $(7)$ by $t(\varphi) \cdot \nu$ and integrating on $\Gamma$, we get

$$
0=\int_{\Gamma}\left(\frac{\partial \mathbf{u}}{\partial \nu}\right)^{2} t(\varphi) \cdot \nu d s=f_{\Gamma_{0}} d s_{0} \varphi \nu_{0} \cdot \int_{\Gamma}\left(\frac{\partial \mathbf{u}}{\partial \nu}\right)^{2} \nu d s
$$

which shows that $\nu_{0} \cdot \int_{\Gamma}\left(\frac{\partial \mathbf{u}}{\partial \nu}\right)^{2} \nu d s=0$. Thus, $F_{1}(\sigma, \psi, \Lambda)=0$.

Now let us assume $F_{1}(\sigma, \psi, \Lambda)=0$. Multiplying both sides by $\nu^{i}, i=1,2$, integrating on $\Gamma$ and using (19), we get

$$
\int_{\Gamma}\left(\frac{\partial \mathbf{u}}{\partial \nu}\right)^{2}\left(\nu \cdot t\left(\nu^{i} \circ T\right)+\sigma \nu^{i}\right) d s=0, \quad i=1,2 .
$$

Thus, we have the system of equations:

$$
\left(\begin{array}{ll}
t_{1}\left(\nu^{1} \circ T\right)+\sigma, & t_{2}\left(\nu^{1} \circ T\right) \\
t_{1}\left(\nu^{2}\right) \circ T, & t_{2}\left(\nu^{2} \circ T\right)+\sigma
\end{array}\right) \cdot\left(\int_{\Gamma}\left(\frac{\partial \mathbf{u}}{\partial \nu}\right)^{2} \nu d s\right)=\left(\begin{array}{l}
0 \\
0
\end{array}\right) .
$$


But the matrix of the above system is invertible for $\psi \in C^{2}\left(\Gamma_{0}\right)$ sufficiently small, because for such $\psi, \nu \circ T$ is near $\nu_{0}=\left(\nu_{0}^{1}, \nu_{0}^{2}\right)$, and thus the matrix of the above system is near

$$
\left(\begin{array}{ll}
t_{1}\left(\nu_{0}^{1}\right)+\sigma, & t_{2}\left(\nu_{0}^{1}\right) \\
t_{1}\left(\nu_{0}^{2}\right), & t_{2}\left(\nu_{0}^{2}\right)+\sigma
\end{array}\right)=\left(\begin{array}{ll}
1 / 2+\sigma, & 0 \\
0, & 1 / 2+\sigma
\end{array}\right)
$$

which is invertible. This proves that the unique solution of the system (20) is the trivial solution. Then, since $\sigma \neq 0$, dividing $F_{1}=0$ by $\sigma$ we obtain (7), which proves the lemma.

Now, we are able to prove Theorem 1.1. The essential tool we use is the implicit function theorem applied to the function $F$ at $(0,0,0)$. Thus, we need to prove that $F$ is of class $C^{1}$ near $(0,0,0)$ and its derivative with respect to $(\psi, \Lambda)$ defines an isomorphism thanks to the additional term mentioned in Remark 2.7 .

3. Proof of the main result. We prove first the following lemma:

Lemma 3.1. The function $F: \mathbb{R} \times C^{2}\left(\Gamma_{0}\right) \times \mathbb{R} \rightarrow C^{0}\left(\Gamma_{0}\right) \times \mathbb{R}$ is of class $C^{1}$ near $(0,0,0)$. Moreover, the derivative of $F$ with respect to $(\psi, \Lambda)$ at $(0,0,0)$ is given by

$$
\begin{aligned}
D_{\psi, \Lambda} F_{1}(0,0,0)(\phi, \lambda) & =-\frac{d^{2} \phi}{d s_{0}^{2}}-\mathcal{H}_{0}^{2} \phi+\lambda+\frac{1}{\left|\Gamma_{0}\right|} \nu_{0} \cdot D^{2} h\left(x_{0}\right) \cdot t(\phi), \\
D_{\psi, \Lambda} F_{2}(0,0,0)(\phi, \lambda) & =\int_{\Gamma_{0}} \phi d s_{0} .
\end{aligned}
$$

Proof. Let us first prove the $C^{1}$ regularity of $F$. For the term $F_{1}$ this can be proved using (17). Indeed, using Lemma 2.4, we have

$$
\begin{aligned}
F_{1}(\sigma, \psi, \Lambda)= & \frac{\mathcal{H} \circ T-\mathcal{H}_{0}}{\sigma}+\Lambda+\frac{2}{\left|\Gamma_{0}\right|} \nu_{0} \cdot D_{\psi} \mathbf{e}(0, \psi)\left(\nu_{0}\right) \\
& -\frac{\sigma}{2}\left(\frac{\partial \mathbf{u}}{\partial \nu}\right)^{2} \circ T
\end{aligned}
$$

where $D_{\psi} \mathbf{e}(0, \psi)\left(\nu_{0}\right)=\left(D_{\psi} \mathbf{e}(0, \psi)\left(\nu_{0}^{1}\right), D_{\psi} \mathbf{e}(0, \psi)\left(\nu_{0}^{2}\right)\right)$. Then, the $C^{1}$ regularity of $F_{1}$ follows from Lemmas 2.1, 2.5 and Corollary 2.3.

The $C^{1}$ regularity of $F_{2}$ (even analytic regularity) follows from the fact that

$$
\mathbf{m}(\sigma, \psi)=\frac{1}{2} \int_{\Gamma_{0}}\left(I+\sigma \psi \nu_{0}\right) \wedge\left(\tau_{0}+\sigma \frac{d\left(\psi \nu_{0}\right)}{d s_{0}}\right) d s_{0} .
$$

Let us now prove (21) and (22). From the equality (23), Lemmas 2.1, 2.5 and Corollary 2.3

$$
D_{\psi, \Lambda} F_{1}(0,0,0)(\phi, \lambda)=-\frac{d^{2} \phi}{d s_{0}^{2}}-\mathcal{H}_{0}^{2} \phi+\lambda+\frac{2}{\left|\Gamma_{0}\right|} \nu_{0} \cdot D_{\psi}^{2} \mathbf{e}(0,0)\left(\nu_{0}, \phi\right)
$$


But from (16) we have

$$
\begin{aligned}
\nu_{0} \cdot D^{2} \psi \mathbf{e}(0,0)\left(\nu_{0}, \phi\right) & =\nu_{0} \cdot\left(D_{\psi}^{2} \mathbf{e}(0,0)\left(\nu_{0}^{1}, \phi\right), D_{\psi}^{2} \mathbf{e}(0,0)\left(\nu_{0}^{2}, \phi\right)\right) \\
& =\nu_{0} \cdot\left(t\left(\nu_{0}^{1}\right), t\left(\nu_{0}^{2}\right)\right) \cdot D^{2} h\left(x_{0}\right) \cdot t(\phi) \\
& =\frac{1}{2} \nu_{0} \cdot D^{2} h\left(x_{0}\right) \cdot t(\phi),
\end{aligned}
$$

which proves (21).

For Eq. (22):

$$
\begin{aligned}
D_{\psi, \Lambda} F_{2}(0,0,0)(\phi, \lambda) & =\lim _{\sigma \rightarrow 0} \frac{D_{\psi, \Lambda} \mathbf{m}(\sigma, 0)(\phi, \lambda)}{\sigma}=\lim _{\sigma \rightarrow 0} \frac{D_{\psi} \mathbf{m}(\sigma, 0)(\phi)}{\sigma} \\
& =\lim _{\sigma \rightarrow 0} \frac{1}{\sigma} \int_{\Gamma_{0}} \sigma \phi d s_{0}=\int_{\Gamma_{0}} \phi d s_{0},
\end{aligned}
$$

which achieves the proof of the lemma.

Proof of Theorem 1.1. As we mentioned in the introduction, the main tool of the proof is the implicit function theorem applied to the function $F$. We have

(i) $F(0,0,0)=0$,

(ii) $F$ is a function of class $C^{1}$ from $\mathbb{R} \times C^{2}\left(\Gamma_{0}\right) \times \mathbb{R}$ near $(0,0,0)$.

If we prove

(iii) $(\phi, \lambda) \in C^{2}\left(\Gamma_{0}\right) \times \mathbb{R} \rightarrow D_{\psi, \Lambda} F(0,0,0)(\phi, \lambda) \in C^{0}\left(\Gamma_{0}\right) \times \mathbb{R}$ defines an isomorphism, then Theorem 1.1 is proved.

Indeed, if (i), (ii) and (iii) are satisfied, from the implicit function theorem, there exists a $\sigma^{*}>0$ and a unique $(\boldsymbol{\psi}, \boldsymbol{\Lambda})$ with

$$
\boldsymbol{\psi} \in C^{1}\left(\left(-\sigma^{*}, \sigma^{*}\right) ; C^{2}\left(\Gamma_{0}\right)\right), \quad \boldsymbol{\Lambda} \in C^{1}\left(\left(-\sigma^{*}, \sigma^{*}\right) ; \mathbb{R}\right),
$$

such that for any $\sigma \in\left(-\sigma^{*}, \sigma^{*}\right)$ we have $F(\sigma, \psi(\sigma), \boldsymbol{\Lambda}(\sigma))=0$. Then, for $\sigma \neq 0$, Lemma 2.8 shows that $\boldsymbol{\psi}(\sigma)$ and $\boldsymbol{\Lambda}(\sigma)$ satisfy Theorem 1.1 .

Thus, it remains only to prove (iii).

(iii.1) Injectivity of $(\phi, \lambda) \rightarrow D_{\psi, \Lambda} F(0,0,0)(\phi, \lambda)$.

Let $(\phi, \lambda) \in C^{2}\left(\Gamma_{0}\right) \times \mathbb{R}$ with $D_{\psi, \Lambda} F(0,0,0)(\phi, \lambda)=0$. Thus $\int_{\Gamma_{0}} \phi d s_{0}=0$. Integrating $D_{\psi, \Lambda} F_{1}(0,0,0)(\phi, \lambda)$ on $\Gamma_{0}$, we get $\lambda=0$. Thus $\phi$ satisfies

$$
\frac{d^{2} \phi}{d s_{0}^{2}}+\mathcal{H}_{0}^{2} \phi=\frac{\nu_{0}}{\left|\Gamma_{0}\right|} \cdot D^{2} h\left(x_{0}\right) \cdot t(\phi) \text { on } \Gamma_{0} .
$$

The only solutions of the previous equation in $\mathbb{R}$ are of the form

$$
\phi\left(s_{0}\right)=C_{1} \cos \left(\mathcal{H}_{0} s_{0}\right)+C_{2} \sin \left(\mathcal{H}_{0} s_{0}\right)+\frac{s_{0}}{4 \pi}\left(\begin{array}{c}
+\sin \left(\mathcal{H}_{0} s_{0}\right) \\
-\cos \left(\mathcal{H}_{0} s_{0}\right)
\end{array}\right) \cdot D^{2} h\left(x_{0}\right) \cdot t(\phi) .
$$

Thus, any solution of (25) is of the previous form and it is $2 \pi r_{0}$-periodic. This implies that $D^{2} h\left(x_{0}\right) \cdot t(\phi)=0$. Since the matrix $D^{2} h\left(x_{0}\right)$ is invertible by virtue of the hypothesis $(6)$, it follows that $t(\phi)=(0,0)$. But $t(\phi)=\left(C_{1}, C_{2}\right) / 2$, which shows that $C_{1}=C_{2}=0$. Thus $(\phi, \lambda)=(0,0)$, which proves (iii.1). 
(iii.2) Surjectivity of $(\phi, \lambda) \rightarrow D_{\psi, \Lambda} F(0,0,0)(\phi, \lambda)$. Let

$$
C_{0}^{2}\left(\Gamma_{0}\right)=\left\{\psi \in C^{2}\left(\Gamma_{0}\right), \int_{\Gamma_{0}} \psi=0\right\}, \quad C_{0}^{0}\left(\Gamma_{0}\right)=\left\{\psi \in C^{0}\left(\Gamma_{0}\right), \int_{\Gamma_{0}} \psi=0\right\} .
$$

First, we prove the surjectivity of

$$
\phi \in C_{0}^{2}\left(\Gamma_{0}\right) \rightarrow D_{\psi, \Lambda} F_{1}(0,0,0)(\phi, 0) \in C_{0}^{0}\left(\Gamma_{0}\right) .
$$

Indeed, let $\Delta^{-1}$ denote the operator $\Delta^{-1}: C_{0}^{0}\left(\Gamma_{0}\right) \rightarrow C_{0}^{2}\left(\Gamma_{0}\right)$, defined by $\Delta^{-1} f=\phi$, such that $\frac{d^{2} \phi}{d s_{0}^{2}}=f$, which is well defined. Then, multiplying by $-\Delta^{-1}$ the equation $D_{\psi, \Lambda} F_{1}(0,0,0)(\phi, 0)=f$, we get that it is equivalent to

$$
\phi+\mathcal{H}_{0}^{2} \Delta^{-1} \phi-\frac{1}{\left|\Gamma_{0}\right|}\left(\Delta^{-1} \nu_{0}\right) \cdot D^{2} h\left(x_{0}\right) \cdot t(\phi)=-\Delta^{-1} f .
$$

Let $A$ denote the operator

$$
A: C_{0}^{2}\left(\Gamma_{0}\right) \rightarrow C_{0}^{2}\left(\Gamma_{0}\right) \text { with } A \phi=\mathcal{H}_{0}^{2} \Delta^{-1} \phi-\frac{1}{\left|\Gamma_{0}\right|}\left(\Delta^{-1} \nu_{0}\right) \cdot D^{2} h\left(x_{0}\right) \cdot t(\phi) .
$$

It is clear that $A$ is compact continuous, and from (iii.1) its eigenvalues are different from -1 . This implies that $I+A$ is continuous invertible. Then, for any $f \in$ $C_{0}^{0}\left(\Gamma_{0}\right)$, there exists $\phi \in C_{0}^{2}\left(\Gamma_{0}\right)$ a solution of $\phi+A \phi=-\Delta^{-1} f$, which is equivalent to $D_{\psi, \Lambda} F_{1}(0,0,0)(\phi, 0)=f$.

Now, let us prove (iii.2). For $(f, \mu) \in C^{0}\left(\Gamma_{0}\right) \times \mathbb{R}$ we can find $(\phi, \lambda) \in C^{2}\left(\Gamma_{0}\right) \times \mathbb{R}$ such that $D_{\psi, \Lambda} F(0,0,0)(\phi, \lambda)=(f, \mu)$. Indeed, if $f_{0}=f-f_{\Gamma_{0}} f$, we have $f_{0} \in C_{0}^{0}\left(\Gamma_{0}\right)$. Thus, there exists a function $\phi_{0} \in C_{0}^{2}\left(\Gamma_{0}\right)$ a solution of $D_{\psi, \Lambda} F_{1}(0,0,0)\left(\phi_{0}, 0\right)=f_{0}$. If we note

$$
\phi=\phi_{0}+\frac{\mu}{\left|\Gamma_{0}\right|}, \quad \lambda=\frac{\mathcal{H}_{0}^{2}}{\left|\Gamma_{0}\right|} \mu+f_{\Gamma_{0}} f_{0} d s_{0},
$$

using the fact that $t(\phi)=t\left(\phi_{0}\right)$, we get $D_{\psi, \Lambda} F(0,0,0)(\phi, \lambda)=(f, \mu)$, which proves the assertion (iii.2).

(iii.3) Continuity of $(\phi, \lambda) \rightarrow D_{\psi, \Lambda} F(0,0,0)(\phi, \lambda)=(f, \mu)$. This follows by the next estimate:

$$
\left\|D_{\psi, \Lambda} F_{1}(0,0,0)(\phi, \lambda)\right\|_{C^{0}\left(\Gamma_{0}\right)}+\left|D_{\psi, \Lambda} F_{2}(0,0,0)(\phi, \lambda)\right| \leq C\left(\|\phi\|_{C^{2}\left(\Gamma_{0}\right)}+|\lambda|\right),
$$

which is a consequence of (21) and (22). Thus (iii) is proved and the proof of the theorem is achieved.

REMARK 3.2. The basic space chosen in this paper is $C^{2}\left(\Gamma_{0}\right)$. Thus, if $(\psi, \Lambda)$ is a solution of $(7),(8)$, then $\Omega(\sigma, \psi)$ is a domain of class $C^{2}$.

In fact, in [5] it is proved that if $\Omega(\sigma, \psi)$ is of class $C^{2}$, then it is analytic.

REMARK 3.3. In this paper we have considered (2), the interior free boundary problem concerning the interior Dirichlet problem (1). Instead of (2), one can consider the following exterior free boundary problem:

$$
\left\{\begin{array}{l}
\frac{1}{2}\left(\frac{\partial u_{\Omega^{*}}}{\partial \nu^{*}}\right)^{2}+\mu \mathcal{H}^{*}+\Lambda^{*}=0 \text { on } \partial \Omega^{*} \\
m\left(\Omega^{*}\right)-m_{0}=0
\end{array}\right.
$$


$u_{\Omega^{*}}$ being the unique variational solution in $\stackrel{\circ}{W}_{0}^{1}\left({ }^{e} \Omega^{*}\right)$, the closure of $\mathcal{D}\left({ }^{e} \Omega^{*}\right)$ for the semi-norm $\|D \varphi\|_{\mathbb{L}^{p}\left(e \Omega^{*}\right)}$, of the exterior Dirichlet problem:

$$
\left\{\begin{array}{rcccc}
-\Delta u_{\Omega^{*}} & = & j & \text { in } & { }^{e} \Omega^{*}:={\overline{\Omega^{*}}}^{c}, \\
u_{\Omega^{*}} & =0 & \text { on } & \partial \Omega^{*} .
\end{array}\right.
$$

Up to now, the sufficient conditions are not known for the existence of a $\left(\Omega^{*}, \Lambda^{*}\right), \Omega^{*}$ regular, solution of $(26)$.

We have found that if $j \in \mathbb{L}^{p}\left(\mathbb{R}^{2}\right), p>2$ is with compact support and

$$
h_{e}(x)=\frac{1}{2} \int_{e_{B\left(x, r_{0}\right)}}\left|D u_{B\left(x, r_{0}\right)}\right|^{2} d x
$$

is such that

$$
D h_{e}(0,0)=0, \quad D^{2} h_{e}(0,0) \quad \text { invertible, }
$$

then we have an existence result for the problem (26) similar to Theorem 1.1. The proof of this result is extremely similar to the proof of Theorem 1.1; so we do not present it here. The only difference with respect to the interior free boundary problem (2) is that we need the differentiability with respect to the shape of the exterior Dirichlet problem (27) instead of (1). More precisely we need results, similar to Lemmas 2.2-2.5 and Corollary 2.3 for the problem (27). The proof of these differentiability results is more technical than the proof of Lemmas 2.2-2.5 and Corollary 2.3. We remark that these results for $p=2$ are presented in $[6]$.

\section{REFERENCES}

[1] J. Descloux, On the two-dimensional magnetic shaping problem without surface tension, École Polytechnique Fédérale de Lausanne, Suisse, 1990

[2] D. Gilbarg and N. S. Trudinger, Elliptic Partial Differential Equations of Second Order, SpringerVerlag, Berlin, 1983

[3] A. Henrot and M. Pierre, About critical points of the energy in an electromagnetic shaping problem, Lecture Notes in Control and Information Sciences, J. P. Zolésio (Ed.), Boundary Control and Boundary Variation, 178, Sophia Antipolis, 1991, pp. 238-252

[4] A. Henrot and M. Pierre, About existence of equilibria in electromagnetic casting, Quarterly of Applied Mathematics, XLIX(3), 563-575 (1991)

[5] A. Henrot and M. Pierre, Un problème inverse en formage des métaux liquides, Mathematical Modelling and Numerical Analysis, 23, 155-177 (1989)

[6] A. Novruzi, Contribution en Optimisation de Formes et Applications, Ph.D. Thesis, Université Henri Poincaré Nancy 1, 1997

[7] J. Simon, Differentiation with respect to the domain in boundary value problems, Numerical Functional Analysis and Optimization, 2(7\&8), 649-687 (1980)

[8] J. Sokolowski and J. P. Zolesio, Introduction to Shape Optimization. Shape Sensitivity Analysis, Springer-Verlag, New York, 1992 